IJMMS 28:10 (2001) 561-570

PII. S0161171201007256

http://ijmms.hindawi.com

(c) Hindawi Publishing Corp.

\title{
NORMAL CHARACTERIZATIONS OF LATTICES
}

\author{
CARMEN D. VLAD
}

(Received 30 March 2001)

\begin{abstract}
Let $\mathbf{X}$ be an arbitrary nonempty set and $\mathscr{L}$ a lattice of subsets of $\mathbf{X}$ such that $\varnothing, \mathbf{X} \in \mathscr{L}$. Let $\mathscr{A}(\mathscr{L})$ denote the algebra generated by $\mathscr{L}$ and $\mathbf{I}(\mathscr{L})$ denote those nontrivial, zero-one valued, finitely additive measures on $\mathscr{A}(\mathscr{L})$. In this paper, we discuss some of the normal characterizations of lattices in terms of the associated lattice regular measures, filters and outer measures. We consider the interplay between normal lattices, regularity or $\sigma$-smoothness properties of measures, lattice topological properties and filter correspondence. Finally, we start a study of slightly, mildly and strongly normal lattices and express then some of these results in terms of the generalized Wallman spaces.
\end{abstract}

2000 Mathematics Subject Classification. 28A12, $28 \mathrm{C} 15$.

1. Introduction. This paper presents a systematic study of various concepts pertaining to normal lattices. Normal lattices are an important class of lattices which have been investigated both in an abstract setting and in a point-set framework by others (see $[1,4,8,9])$.

In Section 2, we give notations and definitions which is fairly standard (see [1, 2, $3,10])$ and a brief background consisting of several results pertaining to lattices, measures, and filters.

In Section 3, we study normality properties of lattices in the point-set framework; most of this material can be readily extended to general lattices. We discuss some of the implications between normality properties and the associated lattice regular measures; in the case of zero-one valued lattice regular measures, these properties have associated filter and topological consequences. Also, by choosing normal lattices in a topological setting, we present interesting results on the associated outer measures on both $\mathscr{L}$ and $\mathscr{L}^{\prime}$.

In Section 4, we start a study of slightly, mildly, and strongly normal lattices and we investigate them from a measure theoretic point of view and thereby extend some results given in $[6,9]$.

This work is then carried out in Section 5 where we express some of these results in terms of generalized Wallman spaces. Specific characteristic on regularity and normality are given for the various Wallman spaces associated with the considered measures of $\mathbf{I}_{\sigma}(\mathscr{L}), \mathbf{I}_{R}^{\sigma}(\mathscr{L})$, and $\mathbf{I}(\sigma, \mathscr{L})$.

2. Background and notation. In this section, we introduce the notation and terminology that will be used throughout the paper. All is fairly standard and we include it for the reader's convenience. 
Let $\mathbf{X}$ be an arbitrary nonempty set and $\mathscr{L}$ a lattice of subsets of $\mathbf{X}$ such that $\varnothing$, $\mathrm{X} \in \mathscr{L}$. Let $\mathscr{A}(\mathscr{L})$ denote the algebra generated by $\mathscr{L} ; \delta(\mathscr{L})$ be the lattice of all countable intersections of sets from $\mathscr{L}$.

DEFINITION 2.1 (lattice terminology). The lattice $\mathscr{L}$ is called: $\delta$-lattice if $\mathscr{L}$ is closed under countable intersections; complement generated if $L \in \mathscr{L}$ implies $L=\cap L_{n}^{\prime}, n=$ $1, \ldots, \infty, L_{n} \in \mathscr{L}$ (where prime denotes the complement); disjunctive if for $x \in \mathbf{X}$ and $L_{1} \in \mathscr{L}$ such that $x \notin L_{1}$ there exists $L_{2} \in \mathscr{L}$ with $x \in L_{2}$ and $L_{1} \cap L_{2}=\varnothing$; separating (or $\mathbf{T}_{1}$ ) if $x, y \in \mathbf{X}$ and $x \neq y$ implies there exists $L \in \mathscr{L}$ such that $x \in L, y \notin L ; \mathbf{T}_{2}$ if for $x, y \in \mathbf{X}$ and $x \neq y$ there exist $L_{1}, L_{2} \in \mathscr{L}$ such that $x \in L_{1}^{\prime}, y \in L_{2}^{\prime}$, and $L_{1}^{\prime} \cap L_{2}^{\prime}=\varnothing$; normal if for any $L_{1}, L_{2} \in \mathscr{L}$ with $L_{1} \cap L_{2}=\varnothing$ there exist $L_{3}, L_{4} \in \mathscr{L}$ with $L_{1} \subset L_{3}^{\prime}, L_{2} \subset L_{4}^{\prime}$, and $L_{3}^{\prime} \cap L_{4}^{\prime}=\varnothing$; compact if for any collection $\left\{L_{\alpha}\right\}$ of sets of $\mathscr{L}$ with $\cap_{\alpha} L_{\alpha}=\varnothing$, there exists a finite subcollection with empty intersection; countably compact if for any countable collection $\left\{L_{\alpha}\right\}$ of sets of $\mathscr{L}$ with $\cap_{\alpha} L_{\alpha}=\varnothing$, there exists a finite subcollection with empty intersection; countably paracompact if for any sequence $\left\{A_{n} \in \mathscr{L}\right\}, A_{n} \downarrow \varnothing$, there exists $\left\{L_{n} \in \mathscr{L}\right\}$, such that $A_{n} \subseteq L_{n}^{\prime}$ and $L_{n}^{\prime} \downarrow \varnothing$; Lindelöf if for any collection $\left\{L_{\alpha}\right\}$ of sets of $\mathscr{L}$ with $\cap_{\alpha} L_{\alpha}=\varnothing$, there exists a countable subcollection with empty intersection.

DEFINITION 2.2 (measure terminology). We denote by $\mathbf{M}(\mathscr{L})$ those nonnegative, finite, finitely additive measures on $\mathscr{A}(\mathscr{L})$. A measure $\mu \in \mathbf{M}(\mathscr{L})$ is called: $\sigma$-smooth on $\mathscr{L}$ if for all sequences $\left\{L_{n}\right\}$ of sets of $\mathscr{L}$ with $L_{n} \downarrow \varnothing, \mu\left(L_{n}\right) \rightarrow 0 ; \sigma$-smooth on $\mathscr{A}(\mathscr{L})$ if for all sequences $\left\{A_{n}\right\}$ of sets of $\mathscr{A}(\mathscr{L})$ with $A_{n} \downarrow \varnothing, \mu\left(A_{n}\right) \rightarrow 0$, that is, countably additive; strongly $\sigma$-smooth on $\mathscr{L}$ if and only if for any sequence $\left\{L_{n} \in \mathscr{L}\right\} L_{n} \downarrow L$ where $L \in \mathscr{L}$, then $\mu(L)=\inf \mu\left(L_{n}\right)=\lim \mu\left(L_{n}\right) ; \mathscr{L}$-regular if for any $A \in \mathscr{A}(\mathscr{L}), \mu(A)=$ $\sup \{\mu(L) \mid L \subset A, L \in \mathscr{L}\}$.

We denote by $\mathbf{M}_{R}(\mathscr{L})$ the set of $\mathscr{L}$-regular measures of $\mathbf{M}(\mathscr{L}) ; \mathbf{M}_{\sigma}(\mathscr{L})$ the set of $\sigma$-smooth measures on $\mathscr{L}$, of $\mathbf{M}(\mathscr{L}) ; \mathbf{M}^{\sigma}(\mathscr{L})$ the set of $\sigma$-smooth measures on $\mathscr{A}(\mathscr{L})$ of $\mathbf{M}(\mathscr{L}) ; \mathbf{M}_{R}^{\sigma}(\mathscr{L})$ the set of $\mathscr{L}$-regular measures of $\mathbf{M}^{\sigma}(\mathscr{L})$; and $\mathbf{M}(\sigma, \mathscr{L})$ the set of strongly $\sigma$-smooth measures on $\mathscr{L}$.

In addition, $\mathbf{I}(\mathscr{L}), \mathbf{I}_{R}(\mathscr{L}), \mathbf{I}_{\sigma}(\mathscr{L}), \mathbf{I}^{\sigma}(\mathscr{L}), \mathbf{I}_{R}^{\sigma}(\mathscr{L})$, and $\mathbf{I}(\sigma, \mathscr{L})$ are the subsets of the corresponding M's which consist of the nontrivial zero-one valued measures.

Finally, $\mathbf{P}(\mathscr{L})=\{\pi$, defined on $\mathscr{L}$, nontrivial, monotone and $\pi(A \cap B)=\pi(A) \pi(B)$, $A, B, \in \mathscr{L}\}$ is the set of all premeasures on $\mathscr{L} ; \mathbf{P}_{\sigma}(\mathscr{L})$ is the set of all premeasures on $\mathscr{L}$ which are $\sigma$-smooth on $\mathscr{L}$.

DEFINITION 2.3 (filters-measures correspondence). A filter in $\mathscr{L}$ is a subset of $\mathscr{L}$, $\mathbf{F}(\mathscr{L})$, satisfying the conditions: $\varnothing \notin \mathbf{F}(\mathscr{L}) ; \mathbf{F}(\mathscr{L})$ is closed under finite intersections; $A \in \mathbf{F}(\mathscr{L}), B \in \mathscr{L}$ and $A \subset B$, then $B \in \mathbf{F}(\mathscr{L})$. An ultrafilter in $\mathscr{L}$ is a maximal filter (relative to the partial order on the collection of filters in $\mathscr{L}$ given by inclusion). An $\mathscr{L}$-filter $\mathbf{F}(\mathscr{L})$ is prime if given $A, B \in \mathscr{L}$ such that $A \cup B \in \mathbf{F}(\mathscr{L})$, then either $A \in \mathbf{F}(\mathscr{L})$ or $B \in \mathbf{F}(\mathscr{L})$.

There exists a one-to-one correspondence between

(i) $\mathscr{L}$-filters $\mathbf{F}(\mathscr{L})$ and elements of $\mathbf{P}(\mathscr{L})$ defined by $\pi(L)=1$ if and only if $L \in \mathbf{F}(\mathscr{L})$;

(ii) $\mathscr{L}$-filters $\mathbf{F}(\mathscr{L})$ with countable intersection property and elements of $\mathbf{P}_{\sigma}(\mathscr{L})=$ $\left\{\pi \in \mathbf{P}(\mathscr{L}) \mid\right.$ if $\pi\left(L_{n}\right)=1$ forall $n$ where $L_{n} \in \mathscr{L}$, then $\left.\cap L_{n} \neq \varnothing\right\}$; 
(iii) prime $\mathscr{L}$-filters and elements of $\mathbf{I}(\mathscr{L})$ given by: for any $\mu \in \mathbf{I}(\mathscr{L})$ associate the prime $\mathscr{L}$-filter $\mathbf{F}(\mathscr{L})=\{A \in \mathscr{L} / \mu(A)=1\}$;

(iv) prime $\mathscr{L}$-filters with countable intersection property and elements of $\mathbf{I}_{\sigma}(\mathscr{L})$;

(v) $\mathscr{L}$-ultrafilters and elements of $\mathbf{I}_{R}(\mathscr{L})$ given by: for any $\mathscr{L}$-ultrafilter $\mathbf{F}(\mathscr{L})$ associate the zero-one valued measure defined on $\mathscr{A}(\mathscr{L})$ by: $\mu(E)=1$ if there exists $A \in \mathbf{F}(\mathscr{L}), A \subset E$, and $\mu(E)=0$ if there exists $A \in \mathbf{F}(\mathscr{L}), A \subset E^{\prime}$;

(vi) $\mathscr{L}$-ultrafilters with countable intersection property and elements of $\mathbf{I}_{R}^{\sigma}(\mathscr{L})$.

DEFINITION 2.4 (lattice-measure correspondence). The support of $\mu \in \mathbf{I}(\mathscr{L})$ is $S(\mu)=\cap\{L \in \mathscr{L} \mid \mu(L)=1\}$.

With this notation and in light of the above correspondences, we now note that: for any $\mu \in \mathbf{I}(\mathscr{L})$, there exists $v \in \mathbf{I}_{R}(\mathscr{L})$ such that $\mu \leq v(\mathscr{L})$ (i.e., $\mu(L) \leq v(L)$ for all $L \in \mathscr{L}$ ). For any $\mu \in \mathbf{I}(\mathscr{L})$, there exists $v \in \mathbf{I}_{R}\left(\mathscr{L}^{\prime}\right)$ such that $\mu \leq\left(\mathscr{L}^{\prime}\right)$. $\mathscr{L}$ is compact if and only if $S(\mu) \neq \varnothing$ for every $\mu \in \mathbf{I}_{R}(\mathscr{L})$ and $\mathscr{L}$ is countably compact if and only if $\mathbf{I}_{R}(\mathscr{L})=\mathbf{I}_{R}^{\sigma}(\mathscr{L}) . \mathscr{L}$ is normal if and only if for each $\mu \in \mathbf{I}(\mathscr{L})$, there exists a unique $v \in \mathbf{I}_{R}(\mathscr{L})$ such that $\mu \leq v(\mathscr{L}) . \mathscr{L}$ is regular if and only if whenever $\mu_{1}, \mu_{2} \in \mathbf{I}(\mathscr{L})$ and $\mu_{1} \leq \mu_{2}(\mathscr{L})$, then $S\left(\mu_{1}\right)=S\left(\mu_{2}\right) . \mathscr{L}$ is replete if and only if for any $\mu \in \mathbf{I}_{R}^{\sigma}(\mathscr{L}), S(\mu) \neq \varnothing$. $\mathscr{L}$ is prime-complete if and only if for any $\mu \in \mathbf{I}_{\sigma}(\mathscr{L}), S(\mu) \neq \varnothing . \mathscr{L}$ is Lindelöf if and only if for any $\pi \in \mathbf{P}_{\sigma}(\mathscr{L}), S(\pi) \neq \varnothing$. Finally, if $\mu_{x}$ is the measure concentrated at $x \in \mathbf{X}$ then $\mu_{x} \in \mathbf{I}_{R}(\mathscr{L})$, for all $x \in \mathbf{X}$ if and only if $\mathscr{L}$ is disjunctive.

For further results and related matters see [2, 3, 4].

3. Normal lattices, filters, and outer measures. In this section, we present a number of theorems on the normality properties of lattices. We consider the interplay between normal lattices, $\mathscr{L}$-regular or $\sigma$-smooth measures, associated outer measures and filters. Many of the results derived in this part are not new. Their inclusion is justified by the necessity of enumerating the known facts in this field and the wish to make the paper self-contained.

THEOREM 3.1. Let $\mathscr{L}$ be a lattice of subsets of $\mathrm{X}$ and let $\mathbf{F}(\mathscr{L})$ be a prime $\mathscr{L}$-filter. Define $\mathbf{G}(\mathscr{L})=\{L \in \mathscr{L} \mid L \cap A \neq \varnothing$ for all $A \in \mathbf{F}(\mathscr{L})\}$. Then $\mathbf{G}(\mathscr{L})$ is a prime $\mathscr{L}$-filter if and only if $\mathscr{L}$ is normal (actually $\mathbf{G}(\mathscr{L})$ is an $\mathscr{L}$-ultrafilter).

Proof. (a) Suppose that $\mathscr{L}$ is normal. Let $L \in \mathbf{G}(\mathscr{L}), B \in \mathscr{L}$ and $L \subset B$. For any $A \in \mathbf{F}(\mathscr{L}), \varnothing \neq L \cap A \subset B \cap A$, so $\mathbf{G}(\mathscr{L})$ is closed under supersets. Let $L_{1}, L_{2} \in \mathbf{G}(\mathscr{L})$ such that $L_{1} \cap L_{2} \notin \mathbf{G}(\mathscr{L})$; then $\left(L_{1} \cap A\right) \cap\left(L_{2} \cap A\right)=\varnothing$ for some $A \in \mathbf{F}(\mathscr{L})$. Since $\mathscr{L}$ is normal, there exist $L_{3}, L_{4} \in \mathscr{L}, L_{1} \cap A \subset L_{3}^{\prime}, L_{2} \cap A \subset L_{4}^{\prime}$, and $L_{3}^{\prime} \cap L_{4}^{\prime}=\left(L_{3} \cup L_{4}\right)^{\prime}=\varnothing$, that is, $L_{3} \cup L_{4}=\mathbf{X}$.

Suppose that $L_{3} \in \mathbf{F}(\mathscr{L})$; then $L_{1} \cap A \cap L_{3}=\varnothing$, with $A, L_{3}, A \cap L_{3} \in \mathbf{F}(\mathscr{L})$; contradiction, since $L_{1} \in \mathbf{G}(\mathscr{L})$. Similarly, for $L_{4} \in \mathbf{F}(\mathscr{L})$. Now, suppose that $L_{1}, L_{2} \in \mathscr{L}$ and $L_{1} \cup L_{2} \in$ $\mathbf{G}(\mathscr{L})$ but $L_{1}, L_{2} \notin \mathbf{G}(\mathscr{L})$. Then there exist $A, B \in \mathbf{F}(\mathscr{L})$ with $L_{1} \cap A=\varnothing, L_{2} \cap B=\varnothing$, and $\left(L_{1} \cup L_{2}\right) \cap(A \cap B)=\varnothing$, contradiction. It follows that $\mathbf{G}(\mathscr{L})$ is a prime $\mathscr{L}$-filter.

If $\mathbf{G}(\mathscr{L}) \subset \mathbf{H}(\mathscr{L})$, where $\mathbf{H}(\mathscr{L})$ is an $\mathscr{L}$-filter and if $L \in \mathbf{H}(\mathscr{L})$, then for any $A \in \mathbf{F}(\mathscr{L}) \subset$ $\mathbf{G}(\mathscr{L}) \subset \mathbf{H}(\mathscr{L}), L \cap A \neq \varnothing$, therefore $\mathbf{G}(\mathscr{L})$ is an $\mathscr{L}$-ultrafilter.

(b) Now let $\mu \in \mathbf{I}(\mathscr{L})$ corresponding to $\mathbf{F}(\mathscr{L})$ and suppose $\mu \leq \nu_{1}, \nu_{2}(\mathscr{L})$ where $v_{1}, v_{2} \in$ $\mathbf{I}_{R}(\mathscr{L})$. Corresponding to $\nu_{1}$ and $\nu_{2}$ we have the $\mathscr{L}$-ultrafilters $\mathbf{F}_{1}(\mathscr{L})$ and $\mathbf{F}_{2}(\mathscr{L})$, with 
$\mathbf{F}(\mathscr{L}) \subset \mathbf{F}_{1}(\mathscr{L}), \mathbf{F}_{2}(\mathscr{L}) \subset \mathbf{G}(\mathscr{L})$, hence $\mathbf{F}_{1}(\mathscr{L})=\mathbf{F}_{2}(\mathscr{L}),=\mathbf{G}(\mathscr{L})$, that is, $v \in \mathbf{I}_{R}(\mathscr{L})$ must be unique, therefore $\mathscr{L}$ is normal.

THEOREM 3.2. Let $\mathscr{L}$ be a lattice of subsets of $\mathrm{X}$ and define

$$
\mathbf{J}(\mathscr{L})=\left\{\pi \in \mathbf{P}(\mathscr{L}) \mid \text { if } \mathbf{X}=L_{1} \cup L_{2} \text {, then } \pi\left(L_{1}\right)=1 \text { or } \pi\left(L_{2}\right)=1, L_{1}, L_{2} \in \mathscr{L}\right\} .
$$

Let $\pi \in \mathrm{J}(\mathscr{L})$ and define

$$
\mathbf{F}(\mathscr{L})=\{A \in \mathscr{L} \mid \pi(A)=1\}, \quad \mathbf{G}(\mathscr{L})=\{L \in \mathscr{L} \mid L \cap A \neq \varnothing \forall A \in \mathbf{F}(\mathscr{L})\} .
$$

If $\mathscr{L}$ is normal then $\mathbf{G}(\mathscr{L})$ is an $\mathscr{L}$-ultrafilter.

Proof. Clearly, F $(\mathscr{L})$ is an $\mathscr{L}$-filter, not a prime $\mathscr{L}$-filter. The proof of the fact that $\mathbf{G}(\mathscr{L})$ is a prime $\mathscr{L}$-filter follows the corresponding proof of Theorem 3.1 since we only need to show $L_{3}^{\prime} \cap L_{4}^{\prime} \neq \varnothing$.

DEFINITION 3.3 (associated outer measures). For $\mu \in \mathbf{I}(\mathscr{L})$ and $E \subset \mathbf{X}$ define

$$
\mu^{\prime}(E)=\inf \left\{\mu\left(L^{\prime}\right) \mid E \subset L^{\prime}, L \in \mathscr{L}\right\} .
$$

For $\mu \in \mathbf{I}_{\sigma}(\mathscr{L})$ and $E \subset \mathbf{X}$ define

$$
\mu^{\prime \prime}(E)=\inf \left\{\sum_{i} \mu\left(L_{i}^{\prime}\right) \mid E \subset \cup_{i} L_{i}^{\prime}, L_{i} \in \mathscr{L}\right\} .
$$

Then $\mu^{\prime}$ is a finitely subadditive outer measure and $\mu^{\prime \prime}$ is a countably additive outer measure.

Furthermore, if $\mu \in \mathbf{I}_{\sigma}(\mathscr{L})$ then

(a) $\mu \leq \mu^{\prime \prime} \leq \mu^{\prime}(\mathscr{L})$.

(b) $\mu^{\prime \prime} \leq \mu^{\prime}=\mu\left(\mathscr{L}^{\prime}\right)$.

(c) If $\mu \in \mathbf{I}_{R}^{\sigma}(\mathscr{L})$ then $\mu=\mu^{\prime \prime}=\mu^{\prime}(\mathscr{L})$ and $\mu^{\prime \prime}=\mu^{\prime}=\mu\left(\mathscr{L}^{\prime}\right)$.

(d) $\mu \in \mathbf{I}_{R}(\mathscr{L})$ if and only if $\mu=\mu^{\prime}(\mathscr{L})$.

Various lattice topological properties have been characterized in terms of the outer measures $\mu^{\prime \prime}$ and $\mu^{\prime}$. We note here Theorem 3.4 without proof (see [7]).

THEOREM 3.4. The lattice $\mathscr{L}$ is normal if and only if for $\nu \in \mathbf{I}(\mathscr{L})$ and $\mu \in \mathbf{I}_{R}(\mathscr{L})$ with $v \leq \mu(\mathscr{L})$, then $\mu^{\prime}=\nu^{\prime}(\mathscr{L})$.

THEOREM 3.5. Let $\mu \in \mathbf{I}_{R}(\mathscr{L})$ and $v \in \mathbf{I}_{R}\left(\mathscr{L}^{\prime}\right)$ such that $\mu \leq v\left(\mathscr{L}^{\prime}\right)$. Then $\mathscr{L}$ is normal if and only if $\mu^{\prime}=v^{\prime}(\mathscr{L})$ for all such $\mu$ and $\nu$.

Proof. Suppose $\mu \leq v\left(\mathscr{L}^{\prime}\right)$. Then $v \leq \mu(\mathscr{L})$ and since $\mu \in \mathbf{I}_{R}(\mathscr{L})$ it follows that $\mu=\mu^{\prime}(\mathscr{L})$. Then $v \leq \mu=\mu^{\prime} \leq \nu^{\prime}(\mathscr{L})$. Suppose $\mathscr{L}$ is normal. Let $A \in \mathscr{L}$ with $\mu(A)=0$. Since $\mu \in \mathbf{I}_{R}(\mathscr{L})$, there exists $L \subset A^{\prime}, L \in \mathscr{L}$ with $\mu(L)=1$. But $A \cap L=\varnothing$ implies that there exist $C^{\prime}, D^{\prime}$ such that $A \subset C^{\prime}, L \subset D^{\prime}, C^{\prime} \cap D^{\prime}=\varnothing, C, D \in \mathscr{L}$. Then we have $A \subset C^{\prime} \subset D \subset L^{\prime}$ and $\nu\left(C^{\prime}\right) \leq \nu(D) \leq \mu(D) \leq \mu\left(L^{\prime}\right)=0$. Then $\nu^{\prime}(A)=0$ and since $A$ was arbitrary in $\mathscr{L}, \mu^{\prime}=v^{\prime}(\mathscr{L})$. Conversely, suppose that $\mu^{\prime}=v^{\prime}(\mathscr{L})$ and let $\mu \in \mathbf{I}(\mathscr{L}), \mu_{1}, \mu_{2} \in \mathbf{I}_{R}(\mathscr{L})$ with $\mu \leq \mu_{1}, \mu_{2}(\mathscr{L})$. But $\mu \leq v \in \mathbf{I}_{R}\left(\mathscr{L}^{\prime}\right)$ on $\mathscr{L}^{\prime}$, so we have $v \leq \mu \leq \mu_{1}, \mu_{2}(\mathscr{L})$. By the assumption, $v^{\prime}=\mu_{1}^{\prime}=\mu_{2}^{\prime}(\mathscr{L})$ and therefore we get that $\mu_{1}=\mu_{1}^{\prime}=\nu^{\prime}=\mu_{2}^{\prime}=\mu_{2}$, that is, $\mathscr{L}$ is normal. 
THEOREM 3.6. Let $\mathscr{L}$ be a normal lattice and let $\mu \in \mathbf{I}_{\sigma}(\mathscr{L})$ and $v \in \mathbf{I}_{R}(\mathscr{L})$ such that $\mu \leq v(\mathscr{L})$. Then $v \in \mathbf{I}_{\sigma}\left(\mathscr{L}^{\prime}\right)$. If $\mathscr{L}$ is also countably paracompact then $v \in \mathbf{I}_{R}^{\sigma}(\mathscr{L})$.

Proof. Let $L_{n}^{\prime} \downarrow \varnothing, L_{n} \in \mathscr{L}$ and suppose $v\left(L_{n}^{\prime}\right)=1$ for all $n$. Since $v \in \mathbf{I}_{R}(\mathscr{L})$, there exist $\tilde{L}_{n} \subset L_{n}^{\prime}, \tilde{L}_{n} \in \mathscr{L}, v\left(\tilde{L}_{n}\right)=1$ for all $n$ and $\tilde{L}_{n} \downarrow \varnothing$. Since $\mathscr{L}$ is normal, therefore there exist $A_{n}^{\prime}, B_{n}^{\prime}$ such that $L_{n} \subset A_{n}^{\prime}, \tilde{L}_{n} \subset B_{n}^{\prime}, A_{n}^{\prime} \cap B_{n}^{\prime}=\varnothing$ and $A_{n}, B_{n} \in \mathscr{L}$. Consider the sequence of inclusions $\tilde{L}_{n} \subset B_{n}^{\prime} \subset A_{n} \subset L_{n}^{\prime}$. Since $L_{n}^{\prime} \downarrow \varnothing$, we may assume, with no loss of generality, that $\tilde{L}_{n}, B_{n}^{\prime}$, and $A_{n} \downarrow \varnothing . \mu \in \mathbf{I}_{\sigma}(\mathscr{L})$ implies $\mu\left(B_{n}^{\prime}\right) \rightarrow 0$. But $\mu\left(B_{n}^{\prime}\right) \geq$ $v\left(B_{n}^{\prime}\right)=1$, contradiction. Next, let $\mathscr{L}$ be also countably paracompact and let $v \in \mathbf{I}_{\sigma}\left(\mathscr{L}^{\prime}\right)$, $B_{n} \in \mathscr{L}, B_{n} \downarrow \varnothing$. There exist $A_{n} \in \mathscr{L}, B_{n} \subset A_{n}^{\prime} \downarrow \varnothing$, and $v\left(B_{n}\right) \leq v\left(A_{n}^{\prime}\right) \rightarrow 0$. Therefore $\nu \in \mathbf{I}_{R}^{\sigma}(\mathscr{L})$.

Consider now the following theorem which is similar to Theorem 3.6 in the case of strongly $\sigma$-smoothness.

THEOREM 3.7. Let $\mathscr{L}$ be a $\delta$ normal lattice and let $\mu \in \mathbf{I}(\sigma, \mathscr{L})$ and $v \in \mathbf{I}_{R}(\mathscr{L})$ such that $\mu \leq v(\mathscr{L})$. Then $v \in \mathbf{I}\left(\sigma, \mathscr{L}^{\prime}\right)$.

Proof. Let $L_{n}^{\prime} \downarrow L^{\prime}, L_{n}, L \in \mathscr{L}$ and suppose that $v\left(L_{n}^{\prime}\right)=1$ for all $n$ but $v\left(L^{\prime}\right)=0$. Then, since $\mathscr{L}$ is normal, it follows that there exist $A_{n} \in \mathscr{L}, A_{n} \subset L_{n}^{\prime}$, and $\mu\left(A_{n}\right)=1$ (see [8]).

Now we may assume that $A_{n} \downarrow A \in \mathscr{L}$, since $\mathscr{L}$ is a $\delta$-lattice and $\mu(A)=1$ since $\mu \in \mathbf{I}(\sigma, \mathscr{L})$. Then $\nu(A)=1$, but $A \subset L^{\prime}$, contradiction.

The next theorem relates the notions of normal lattice, outer measure and filter.

THEOREM 3.8. The lattice $\mathscr{L}$ is normal if and only if for each $\mu \in \mathbf{I}(\mathscr{L}), \mu^{\prime}$ determines a prime $\mathscr{L}$-filter.

Proof. Let $\mathbf{F}(\mathscr{L})=\left\{L \in \mathscr{L} \mid \mu^{\prime}(L)=1\right\}$. Suppose that $\mathscr{L}$ is normal. Clearly, $\varnothing \notin \mathbf{F}(\mathscr{L})$. Let $L \in \mathbf{F}(\mathscr{L}), B \in \mathscr{L}$ with $L \subset B$; then $1=\mu^{\prime}(L) \leq \mu^{\prime}(B)$, so $B \in \mathbf{F}(\mathscr{L})$. Let $L_{1}, L_{2} \in \mathbf{F}(\mathscr{L})$ but $L_{1} \cap L_{2} \notin \mathbf{F}(\mathscr{L})$. Then $\mu^{\prime}\left(L_{1} \cap L_{2}\right)=0$, that is, there exists $L^{\prime} \supset L_{1} \cap L_{2}, L \in \mathscr{L}$ with $\mu\left(L^{\prime}\right)=0$. We have $L \subset L_{1}^{\prime} \cup L_{2}^{\prime}$ and since $\mathscr{L}$ is normal it follows that $L=L_{3} \cup L_{4}, L_{3} \subset L_{1}^{\prime}$, and $L_{4} \subset L_{2}^{\prime}$. Hence $\mu\left(L_{3}^{\prime}\right)=0$ or $\mu\left(L_{4}^{\prime}\right)=0$, contradiction. Now let $L_{1}, L_{2} \in \mathscr{L}$ such that $L_{1} \cup L_{2} \in \mathbf{F}(\mathscr{L})$ but $L_{1}, L_{2} \notin \mathbf{F}(\mathscr{L})$, that is, $\mu^{\prime}\left(L_{1} \cup L_{2}\right)=1$ but $\mu\left(L_{1}^{\prime}\right)=\mu\left(L_{2}^{\prime}\right)=0$. We get a contradiction, since $\mu^{\prime}\left(L_{1} \cup L_{2}\right) \leq \mu\left(L_{1}^{\prime}\right)+\mu\left(L_{2}^{\prime}\right)$.

Conversely, suppose that $\mu^{\prime}$ determines a prime $\mathscr{L}$-filter, $\mathbf{F}(\mathscr{L})$. If $\mathscr{L}$ is not normal, then there exist $\mu \in \mathbf{I}(\mathscr{L}), \mu_{1}, \mu_{2} \in \mathbf{I}_{R}(\mathscr{L}), \mu_{1} \neq \mu_{2}$ and $\mu \leq \mu_{1}, \mu_{2} \leq \mu^{\prime}(\mathscr{L})$. To $\mu_{1}$ and $\mu_{2}$ correspond the $\mathscr{L}$-ultrafilters $\mathbf{G}(\mathscr{L})$ and $\mathbf{H}(\mathscr{L})$ and by the above we have $\mathbf{G}(\mathscr{L}), \mathbf{H}(\mathscr{L}) \subset$ $\mathbf{F}(\mathscr{L})$; therefore $\mathbf{G}(\mathscr{L})=\mathbf{H}(\mathscr{L})=\mathbf{F}(\mathscr{L})$, which implies $\mu_{1}=\mu_{2}$ which is a contradiction.

The following theorem on the equality of two outer measures $\mu^{\prime \prime}$ and $\mu^{\prime}$ depending on the normality of $\mathscr{L}$ is well known (see [7]) and we just state it without proof.

THEOREM 3.9. Let $\mu \in \mathbf{I}_{\sigma}(\mathscr{L})$, then $\mu^{\prime \prime}=\mu^{\prime}(\mathscr{L})$ if one of the following conditions is satisfied:

(a) $\mathscr{L}$ is normal and $\delta$-lattice; or

(b) $\mathscr{L}$ is normal and countably paracompact. 
REMARK 3.10. Note that if Theorem 3.9(b) is true, then by Theorem 3.6, for $\mu \in$ $\mathbf{I}_{\sigma}(\mathscr{L})$ and $v \in \mathbf{I}_{R}(\mathscr{L})$ such that $\mu \leq v(\mathscr{L})$ it follows that $v \in \mathbf{I}_{R}^{\sigma}(\mathscr{L})$ and then by Definition 3.3(c) we have $v=v^{\prime}=v^{\prime \prime}(\mathscr{L})$. Therefore in this case, for both $\mu \in \mathbf{I}_{\sigma}(\mathscr{L})$ and the dominating $\nu \in \mathbf{I}_{R}(\mathscr{L})$ we have the equality of $\mu^{\prime \prime}, \mu^{\prime}$ and $\nu^{\prime}, v^{\prime \prime}$, respectively.

4. Normal, slightly normal, mildly normal, and strongly normal lattices. In this section, we summarize some known results (see $[6,9]$ ) on normal, slightly normal, mildly normal, and strongly normal lattices and give slightly different proofs. First, we recall some definitions.

DeFinItion 4.1. The lattice $\mathscr{L}$ is slightly normal if for all $\mu \in \mathbf{I}_{\sigma}\left(\mathscr{L}^{\prime}\right)$, there exists a unique $v \in \mathbf{I}_{R}(\mathscr{L})$ such that $\mu \leq v(\mathscr{L})$.

Definition 4.2. The lattice $\mathscr{L}$ is mildly normal if for all $\mu \in \mathbf{I}_{\sigma}(\mathscr{L})$, there exists a unique $v \in \mathbf{I}_{R}(\mathscr{L})$ such that $\mu \leq v(\mathscr{L})$.

THEOREM 4.3. If $\mathscr{L}$ is regular and Lindelöf then it is mildly and slightly normal.

Proof. Suppose there exist $\mu \in \mathbf{I}_{\sigma}(\mathscr{L}), v_{1}, v_{2} \in \mathbf{I}_{R}(\mathscr{L})$ such that $v_{1} \neq v_{2}$ and $\mu \leq$ $v_{1}, \nu_{2}(\mathscr{L})$. Then there exist $L_{1}, L_{2} \in \mathscr{L}$ such that $L_{1} \cap L_{2}=\varnothing, v_{1}\left(L_{1}\right)=v_{2}\left(L_{2}\right)=1$, and $\nu_{1}\left(L_{2}\right)=\nu_{2}\left(L_{1}\right)=0$. Since $\mathscr{L}$ is regular, $S(\mu)=S\left(\nu_{1}\right) \subset L_{1}$ and $S(\mu)=S\left(\nu_{2}\right) \subset L_{2}$, therefore $S(\mu) \subset\left(L_{1} \cap L_{2}\right)=\varnothing$. But $S(\mu)=S\left(\nu_{1}\right)=S\left(\nu_{2}\right)=\cap_{\alpha}\left\{L_{\alpha} \mid \mu\left(L_{\alpha}\right)=1\right\}=\varnothing$. The lattice $\mathscr{L}$ is Lindelöf, hence there exist a countable subcollection $\left\{L_{i}\right\}$ with $\cap_{i}\left\{L_{i} \mid\right.$ $\left.\mu\left(L_{i}\right)=1\right\}=\varnothing$ which give a contradiction since $\mu \in \mathbf{I}_{\sigma}(\mathscr{L})$. Hence $\mathscr{L}$ is mildly normal.

Next, let $\mu \in \mathbf{I}_{\sigma}\left(\mathscr{L}^{\prime}\right), v_{1}, v_{2}, \in \mathbf{I}_{R}(\mathscr{L})$ such that $v_{1} \neq v_{2}$ and $\mu \leq v_{1}, v_{2}(\mathscr{L})$ as before. Then $S(\mu)=\varnothing$. Let $\pi(L)=\sup \left\{\mu\left(\tilde{L}^{\prime}\right) \mid \tilde{L}^{\prime} \subset L, L \in \mathscr{L}\right\}, \pi \in \mathbf{P}(\mathscr{L})$ and $S(\mu)=S(\pi)$ since $\mathscr{L}$ is regular. But $\mu \in \mathbf{I}_{\sigma}\left(\mathscr{L}^{\prime}\right)$ implies $\pi \in \mathbf{P}_{\sigma}(\mathscr{L})$ and since $\mathscr{L}$ is Lindelöf, $S(\pi) \neq \varnothing$. Then $S(\mu) \neq \varnothing$ which gives a contradiction. It follows that $\mathscr{L}$ is slightly normal.

Now we formulate a series of results omitting their proofs (see [6]). They will be used in the next section.

THEOREM 4.4. (a) If $\mathscr{L}$ is almost countably compact and mildly normal, then $\mathscr{L}$ is normal.

(b) If $\mathscr{L}$ is complement generated, then $\mathscr{L}$ is slightly normal.

DEFINITION 4.5. A lattice $\mathscr{L}$ is called strongly normal if for $\mu, \mu_{1}, \mu_{2} \in \mathbf{I}(\mathscr{L})$ with $\mu \leq \mu_{1}, \mu_{2}(\mathscr{L})$ we have $\mu_{1} \leq \mu_{2}(\mathscr{L})$ or $\mu_{2} \leq \mu_{1}(\mathscr{L})$.

THEOREM 4.6. Any strongly normal lattice $\mathscr{L}$ is normal.

Proof. Suppose that there exists a $\mu \in \mathbf{I}(\mathscr{L})$ such that $\mu \leq v_{1}, v_{2}(\mathscr{L})$ where $v_{1}, v_{2}$ $\in \mathbf{I}_{R}(\mathscr{L})$. Since $\mathscr{L}$ is strongly normal $v_{1} \leq v_{2}(\mathscr{L})$ or $v_{2} \leq v_{1}(\mathscr{L})$. The regular measures $v_{1}$ and $v_{2}$ correspond to $\mathscr{L}$-ultrafilters $\mathbf{F}_{1}(\mathscr{L}), \mathbf{F}_{2}(\mathscr{L})$ with $\mathbf{F}_{1}(\mathscr{L}) \subseteq \mathbf{F}_{2}(\mathscr{L})$ or $\mathbf{F}_{2}(\mathscr{L}) \subseteq \mathbf{F}_{1}(\mathscr{L})$ which is a contradiction. Therefore $\mathscr{L}$ is normal.

THEOREM 4.7. The lattice $\mathscr{L}$ is strongly normal if and only if $\mathbf{I}(\mathscr{L})=\mathbf{J}(\mathscr{L})$.

Proof. Suppose that $\mathbf{I}(\mathscr{L})=\mathbf{J}(\mathscr{L})$ but for arbitrary $\mu, \mu_{1}, \mu_{2} \in \mathbf{I}(\mathscr{L})$ with $\mu \leq \mu_{1}$, $\mu_{2}(\mathscr{L})$ we have $\mu_{1} \nless \mu_{2}(\mathscr{L})$ and $\mu_{2} \nless \mu_{1}(\mathscr{L})$. Then $\mu \leq \mu_{1} \wedge \mu_{2}(\mathscr{L})$ where $\mu_{1} \wedge \mu_{2} \in$ $\mathbf{P}(\mathscr{L})$, but $\notin \mathbf{I}(\mathscr{L})$. Clearly, since $\mathbf{I}(\mathscr{L})=\mathbf{J}(\mathscr{L}), \mu_{1} \wedge \mu_{2} \in \mathbf{I}(\mathscr{L})$ which is a contradiction. 
Conversely, suppose that $\mathscr{L}$ is strongly normal and let $\pi \in \mathbf{J}(\mathscr{L})$. We have then $v \leq$ $\pi=\wedge \mu_{\alpha}(\mathscr{L})$, where $\pi \leq \mu_{\alpha}$ and $\nu, \mu_{\alpha} \in \mathbf{I}(\mathscr{L})$. But $\left\{\mu_{\alpha}\right\}_{\alpha \in \Lambda}$ is totally ordered since $\mathscr{L}$ is strongly normal. Therefore, for any $\alpha, \beta \in \Lambda, v_{\alpha} \leq v_{\beta}(\mathscr{L})$ or $v_{\beta} \leq v_{\alpha}(\mathscr{L})$. Suppose that $\pi\left(L_{1} \cup L_{2}\right)=1, L_{1}, L_{2} \in \mathscr{L}$. Then $\mu_{\alpha}\left(L_{1} \cup L_{2}\right)=1$ for all $\alpha \in \Lambda$. Suppose that for some $\lambda \in \Lambda, \mu_{\lambda}\left(L_{1}\right)=0$; then $\mu_{\gamma}\left(L_{1}\right)=0$ for all $\mu_{\gamma} \leq \mu_{\lambda}$ hence $\mu_{\gamma}\left(L_{2}\right)=1$ for all $\mu_{\gamma} \leq \mu_{\lambda}(\mathscr{L})$. But also $\mu_{\beta}\left(L_{2}\right)=1$ for all $\mu_{\beta} \geq \mu_{\lambda}(\mathscr{L})$. Thus $\mu_{\alpha}\left(L_{2}\right)=1$ for all $\alpha \in \Lambda$, so $\pi\left(L_{2}\right)=1$ which proves that $\pi \in \mathbf{I}(\mathscr{L})$.

5. Application to the Wallman spaces. Next, we briefly summarize some facts (see [4]) about the Wallman space $\mathbf{I}_{R}^{\sigma}(\mathscr{L})$ and then proceed to consider some relations between the measures and the induced measures on the Wallman spaces. A few of the properties to be considered have been investigated in [5], we give slightly different proofs, and include some of them for completeness. Specific characterizations are given for the various Wallman spaces associated with the considered zero-one valued measures concerning normality and related questions studied in the previous section.

DEFINITION 5.1 (general Wallman spaces and Wallman topologies). The Wallman topology in $\mathbf{I}_{R}^{\sigma}(\mathscr{L})$ is obtained by taking all

$$
W_{\sigma}(L)=\left\{\mu \in \mathbf{I}_{R}^{\sigma}(\mathscr{L}) \mid \mu(L)=1\right\}, \quad L \in \mathscr{L},
$$

as a base for the closed sets in $\mathbf{I}_{R}^{\sigma}(\mathscr{L})$ and then $\mathbf{I}_{R}^{\sigma}(\mathscr{L})$ is called the general Wallman space associated with $\mathbf{X}$ and $\mathscr{L}$. Assuming $\mathscr{L}$ is disjunctive, $W_{\sigma}(\mathscr{L})=\left\{W_{\sigma}(L) \mid L \in \mathscr{L}\right\}$ is a lattice in $\mathbf{I}_{R}^{\sigma}(\mathscr{L})$, isomorphic to $\mathscr{L}$ under the map $L \rightarrow W_{\sigma}(L), L \in \mathscr{L} . W_{\sigma}(\mathscr{L})$ is replete and a base for the closed sets $t W_{\sigma}(\mathscr{L})$, all arbitrary intersections of sets of $W_{\sigma}(\mathscr{L})$. It is this topological space $\left[\mathbf{I}_{R}^{\sigma}(\mathscr{L}), t W_{\sigma}(\mathscr{L})\right]$ and lattice $W_{\sigma}(\mathscr{L})$ which we will consider here and in subsequent sections.

Analogously, we also consider $\mathbf{I}_{\sigma}(\mathscr{L})$ and $V_{\sigma}(\mathscr{L})=\left\{V_{\sigma}(L) \mid L \in \mathscr{L}\right\}$ where $V_{\sigma}(A)=$ $\left\{\mu \in \mathbf{I}_{\sigma}(\mathscr{L}) \mid \mu(A)=1\right\}, A \in \mathscr{A}(\mathscr{L})$. Note that here we do not need the assumption of disjunctiveness on $\mathscr{L}$ and that $V_{\sigma}(\mathscr{L})$ is prime complete and a base for the closed sets $t V_{\sigma}(\mathscr{L})$ of $\mathbf{I}_{\sigma}(\mathscr{L})$. Note also that in a similar way one can construct the Wallman topological spaces $\left[\mathbf{I}_{R}(\mathscr{L}), t W(\mathscr{L})\right]$, (here $\mathscr{L}$ must be separating, too) and $[\mathbf{I}(\mathscr{L}), t V(\mathscr{L})]$.

If $A \in \mathscr{A}(\mathscr{L})$, then $W_{\sigma}(A)=\left\{\mu \in \mathbf{I}_{R}^{\sigma}(\mathscr{L}) \mid \mu(A)=1\right\}$ and the following statements are true:

$$
\begin{gathered}
W_{\sigma}(A \cup B)=W_{\sigma}(A) \cup W_{\sigma}(B), \\
W_{\sigma}(A \cap B)=W_{\sigma}(A) \cap W_{\sigma}(B), \\
W_{\sigma}\left(A^{\prime}\right)=W_{\sigma}(A)^{\prime}, \\
A \supset B \quad \text { iff } W_{\sigma}(A) \supset W_{\sigma}(B), \\
\mathscr{A}\left(W_{\sigma}(\mathscr{L})\right)=W_{\sigma}(\mathscr{A}(\mathscr{L})) .
\end{gathered}
$$

DEFINITION 5.2 (the induced measure). Let $\mu \in \mathbf{I}_{R}^{\sigma}(\mathscr{L})$ and consider the induced measure $\underline{\mu} \in \mathbf{I}_{R}^{\sigma}\left(W_{\sigma}(\mathscr{L})\right)$, defined by

$$
\underline{\mu}\left(W_{\sigma}(A)\right)=\mu(A), \quad A \in \mathscr{A}(\mathscr{L}) .
$$

The map $\mu \rightarrow \underline{\mu}$ is a bijection between $\mathbf{I}_{R}^{\sigma}(\mathscr{L})$ and $\mathbf{I}_{R}^{\sigma}\left(W_{\sigma}(\mathscr{L})\right.$ ). 
Theorem 5.3. (a) Consider $\mathbf{I}_{R}^{\sigma}(\mathscr{L})$ and $W_{\sigma}(\mathscr{L})$ with $\mathscr{L}$ disjunctive. Then $W_{\sigma}(\mathscr{L})$ is regular if and only if for all $\mu_{1}, \mu_{2} \in \mathbf{I}(\mathscr{L})$ and $\nu \in \mathbf{I}_{R}^{\sigma}(\mathscr{L})$, if $\mu_{1} \leq \mu_{2}, \nu(\mathscr{L})$ then $\mu_{2} \leq$ $v(\mathscr{L})$.

(b) Consider $\mathbf{I}_{\sigma}(\mathscr{L})$ and $V_{\sigma}(\mathscr{L})$. Then $V_{\sigma}(\mathscr{L})$ is regular if and only if for all $\mu_{1}, \mu_{2} \in$ $\mathbf{I}(\mathscr{L})$ and $v \in \mathbf{I}_{\sigma}(\mathscr{L})$, if $\mu_{1} \leq \mu_{2}(\mathscr{L})$ and $\mu_{1} \leq v(\mathscr{L})$ then $\mu_{2} \leq v(\mathscr{L})$.

Proof. (a) Let $\mu_{1}, \mu_{2} \in \mathbf{I}(\mathscr{L})$ such that $\mu_{1} \leq \mu_{2}(\mathscr{L})$, there exist $\underline{\mu}_{1}, \underline{\mu}_{2} \in \mathbf{I}\left(W_{\sigma}(\mathscr{L})\right)$ and $\underline{\mu}_{1}\left(W_{\sigma}(L)\right)=\mu_{1}(L), \underline{\mu}_{2}\left(W_{\sigma}(L)\right)=\mu_{2}(L)$ for all $L \in \mathscr{L}$. Moreover, $\mu_{1}(L) \leq \mu_{2}(L)$ implies $\underline{\mu}_{1} \leq \underline{\mu}_{2}$ on $W_{\sigma}(\mathscr{L})$. Suppose $W_{\sigma}(\mathscr{L})$ is regular, then $S\left(\underline{\mu}_{1}\right)=S\left(\underline{\mu}_{2}\right)$, where $S\left(\underline{\mu}_{1}\right)=$ $\cap\left\{W_{\sigma}(L) \in W_{\sigma}(\mathscr{L}) \mid \underline{\mu}_{1}\left(W_{\sigma}(L)\right)=1, L \in \mathscr{L}\right\}$. Let now $v \in \mathbf{I}_{R}^{\sigma}(\mathscr{L})$ with $\mu_{1} \leq v(\mathscr{L})$. We have $\underline{v} \in \mathbf{I}_{R}^{\sigma}\left(W_{\sigma}(\mathscr{L})\right)$ and $\underline{\mu}_{1} \leq \underline{v}\left(W_{\sigma}(\mathscr{L})\right)$ therefore $S(\underline{v}) \subset S\left(\underline{\mu}_{1}\right)=S\left(\underline{\mu}_{2}\right)$; hence $\underline{\mu}_{2} \leq$ $\underline{v}\left(W_{\sigma}(\mathscr{L})\right)$, that is, $\mu_{2} \leq v(\mathscr{L})$.

Conversely, let $\mu_{1}, \mu_{2} \in \mathbf{I}(\mathscr{L})$ and $\nu \in \mathbf{I}_{R}^{\sigma}(\mathscr{L})$ such that if $\mu_{1} \leq \mu_{2}(\mathscr{L})$ and $\mu_{1} \leq \nu(\mathscr{L})$ then $\mu_{2} \leq v(\mathscr{L})$. Let now $\lambda_{1}, \lambda_{2} \in \mathbf{I}\left(W_{\sigma}(\mathscr{L})\right)$ and assume $\lambda_{1} \leq \lambda_{2}\left(W_{\sigma}(\mathscr{L})\right)$. Then $\lambda_{1}=\underline{\mu}_{1}$ and $\lambda_{2}=\underline{\mu}_{2}$ where $\mu_{1}, \mu_{2} \in \mathbf{I}(\mathscr{L})$ and $\underline{\mu}_{1} \leq \underline{\mu}_{2}\left(W_{\sigma}(\mathscr{L})\right)$, that is, $\mu_{1} \leq \mu_{2}(\mathscr{L})$. Now $S\left(\underline{\mu}_{2}\right) \subset$ $S\left(\underline{\mu}_{1}\right)$; if $\bar{\lambda} \in S\left(\underline{\mu}_{1}\right)$, then clearly $\lambda \in \mathbf{I}_{R}^{\sigma}(\mathscr{L})$ and $\mu_{1} \leq \lambda(\mathscr{L})$. Hence, by the assumption $\mu_{2} \leq \lambda(\mathscr{L})$ which implies $\lambda \in S\left(\underline{\mu}_{2}\right)$.

Clearly, the proof of (b) is similar and we omit it.

THEOREM 5.4. Consider $\mathbf{I}_{\sigma}(\mathscr{L})$ and $V_{\sigma}(\mathscr{L})$. Then $V_{\sigma}(\mathscr{L})$ is regular if and only if $\mathbf{I}_{\sigma}(\mathscr{L})=\mathbf{I}_{R}^{\sigma}(\mathscr{L})$. (We note here that the assumption of regularity for $V_{\sigma}(\mathscr{L})$ in $\mathbf{I}_{\sigma}(\mathscr{L})$ is very strong.)

Proof. Suppose $\mathbf{I}_{\sigma}(\mathscr{L})=\mathbf{I}_{R}^{\sigma}(\mathscr{L})$. Then $V_{\sigma}(\mathscr{L})=W_{\sigma}(\mathscr{L})$. Now let $\mu_{1}, \mu_{2} \in \mathbf{I}(\mathscr{L}), v \in$ $\mathbf{I}_{\sigma}(\mathscr{L})$, and $\mu_{1} \leq \mu_{2}(\mathscr{L}), \mu_{1} \leq v(\mathscr{L})$. Then, since $\mathbf{I}_{\sigma}(\mathscr{L})=\mathbf{I}_{R}^{\sigma}(\mathscr{L}), \mu_{1} \in \mathbf{I}_{R}^{\sigma}(\mathscr{L})$ so $\mu_{1}=\mu_{2}$ and $\mu_{1}=v$. Conversely, suppose $V_{\sigma}(\mathscr{L})$ is regular and let $\mu \in \mathbf{I}_{\sigma}(\mathscr{L})$, there exists $v \in \mathbf{I}_{R}(\mathscr{L})$ such that $\mu \leq v(\mathscr{L})$, that is, $\underline{\mu} \leq \underline{v}\left(V_{\sigma}(\mathscr{L})\right)$, where $\underline{\mu}, \underline{v} \in \mathbf{I}_{\sigma}\left(V_{\sigma}(\mathscr{L})\right)$. But $S(\underline{\mu})=S(\underline{\nu})$ since $V_{\sigma}(\mathscr{L})$ is regular. Hence $\mu \in S(\underline{\nu})$, that is, $\nu \leq \mu(\mathscr{L})$. It follows that $\mu=\nu$ and then $\mu \in \mathbf{I}_{R}^{\sigma}(\mathscr{L})$.

DEFINITION 5.5. The lattice $\mathscr{L}$ is almost countably compact if $\mu \in \mathbf{I}_{R}\left(\mathscr{L}^{\prime}\right)$ implies $\mu \in \mathbf{I}_{\sigma}(\mathscr{L})$.

THEOREM 5.6. Suppose $\mathscr{L}$ is disjunctive, then we have the following:

(a) Consider $\mathbf{I}_{R}^{\sigma}(\mathscr{L})$ and $W_{\sigma}(\mathscr{L})$ and suppose $\mathscr{L}$ is Lindelöf and satisfies the condition that for all $\mu_{1}, \mu_{2} \in \mathbf{I}(\mathscr{L})$ and $v \in \mathbf{I}_{R}^{\sigma}(\mathscr{L})$, if $\mu_{1} \leq \mu_{2}, v(\mathscr{L})$ then $\mu_{2} \leq v(\mathscr{L})$.

Then $W_{\sigma}(\mathscr{L})$ is slightly and mildly normal.

(b) If $\mathscr{L}$ is complement generated then $W_{\sigma}(\mathscr{L})$ is slightly normal.

(c) If $\mathscr{L}$ is almost countably compact and mildly normal then $W_{\sigma}(\mathscr{L})$ is normal.

Proof. (a) $\mathscr{L}$ disjunctive and Lindelöf implies $W_{\sigma}(\mathscr{L})$ is Lindelöf. Also, by Theorem 5.3 it follows that $W_{\sigma}(\mathscr{L})$ is regular. Now use Theorem 4.3 to conclude that $W_{\sigma}(\mathscr{L})$ is slightly and mildly normal.

(b) $\mathscr{L}$ is complement generated implies $L=\cap_{n} L_{n}{ }^{\prime}, L$ and $L_{n} \in \mathscr{L}$, for all $n$.

$W_{\sigma}(L)=W_{\sigma}\left(\cap_{n} L_{n}{ }^{\prime}\right)=\cap_{n} W_{\sigma}\left(L_{n}{ }^{\prime}\right)=\cap_{n}\left[W_{\sigma}\left(L_{n}\right)\right]^{\prime}$. Hence $W_{\sigma}(\mathscr{L})$ complement generated which implies that $W_{\sigma}(\mathscr{L})$ is slightly normal (by Theorem 4.4).

(c) By the assumption, for any $\mu \in \mathbf{I}_{R}\left(\mathscr{L}^{\prime}\right)$ it follows that $\mu \in \mathbf{I}_{\sigma}(\mathscr{L})$ and then there exists a unique $v \in \mathbf{I}_{R}(\mathscr{L})$ such that $\mu \leq \nu(\mathscr{L})$. Let $\mu \in \mathbf{I}(\mathscr{L})$ such that $\mu \leq \lambda\left(\mathscr{L}^{\prime}\right)$ with 
$\lambda \in \mathbf{I}_{R}\left(\mathscr{L}^{\prime}\right)$. Also $\lambda \in \mathbf{I}_{\sigma}(\mathscr{L})$ and $\lambda \leq \mu \leq v_{1}(\mathscr{L})$ with $v_{1} \in \mathbf{I}_{R}(\mathscr{L})$, unique. Therefore if $\mu \leq \nu_{2}(\mathscr{L})$ with $v_{2} \in \mathbf{I}_{R}(\mathscr{L})$, then $\lambda \leq \mu \leq v_{2}(\mathscr{L})$, and so $v_{1}=v_{2}$.

Hence $\mathscr{L}$ is normal and also $W_{\sigma}(\mathscr{L})$ is normal.

As an immediate consequence, we get the following condition on $V_{\sigma}(\mathscr{L})$ that will be used later on, for Theorem 5.11.

COROLlary 5.7. Consider $\mathbf{I}_{\sigma}(\mathscr{L})$ and $V_{\sigma}(\mathscr{L})$ with $\mathscr{L}$ Lindelöf. If for all $\mu_{1}, \mu_{2} \in \mathbf{I}(\mathscr{L})$ and $v \in \mathbf{I}_{\sigma}(\mathscr{L})$ such that if $\mu_{1} \leq \mu_{2}(\mathscr{L})$ and $\mu_{1} \leq v(\mathscr{L})$, it follows that $\mu_{2} \leq v(\mathscr{L})$, then $V_{\sigma}(\mathscr{L})$ is slightly and mildly normal.

THEOREM 5.8. (a) Let $\mathscr{L}$ be disjunctive, almost countably compact, and mildly normal and let $W_{\sigma}(\mathscr{L})$ be prime complete. Then $\mathscr{L}$ is countably compact.

(b) Let $\mathscr{L}$ be disjunctive, regular, Lindelöf, almost countably compact and let $W_{\sigma}(\mathscr{L})$ be prime complete. Then $\mathscr{L}$ is countably compact.

Proof. (a) We must show that $\mathbf{I}_{R}(\mathscr{L})=\mathbf{I}_{R}^{\sigma}(\mathscr{L})$. Let $\mu \in \mathbf{I}_{R}(\mathscr{L})$; we have $\mu \leq v\left(\mathscr{L}^{\prime}\right)$ where $v \in \mathbf{I}_{R}\left(\mathscr{L}^{\prime}\right)$. Since $\mathscr{L}$ is almost countably compact we have $v \leq \mu(\mathscr{L})$ with $v \in$ $\mathbf{I}_{\sigma}(\mathscr{L})$. But $W_{\sigma}(\mathscr{L})$ is prime complete and by Theorem 5.3 there exists $\lambda \in \mathbf{I}_{R}^{\sigma}(\mathscr{L})$ such that $v \leq \lambda(\mathscr{L}) . \mathscr{L}$ almost countably compact and mildly normal implies that $\mathscr{L}$ is normal (by Theorem 4.4). By the normality of $\mathscr{L}$, the $\mathscr{L}$-regular measure $\mu$ such that $\nu \leq \mu$ must be unique, hence $\mu=\lambda \in \mathbf{I}_{R}^{\sigma}(\mathscr{L})$.

(b) $\mathscr{L}$ regular and Lindelöf implies $\mathscr{L}$ mildly normal and by the above it follows that $\mathscr{L}$ is countably compact.

Finally, we consider another general Wallman topological space, consisting of strongly $\sigma$-smooth measures and analyze the relevant lattice in terms of normality:

$$
[\mathbf{I}(\sigma, \mathscr{L}), t V(\sigma, \mathscr{L})]
$$

where

$$
V(\sigma, \mathscr{L})=\{V(\sigma, L) \mid L \in \mathscr{L}\} \quad \text { with } \quad V(\sigma, L)=\{\mu \in \mathbf{I}(\sigma, \mathscr{L}) \mid \mu(L)=1, L \in \mathscr{L}\} .
$$

We recall a few statements on $\sigma$-smoothness that will be used throughout this section for the reader's convenience (see [7]).

(a) $\mathbf{I}^{\sigma}(\mathscr{L}) \subset \mathbf{I}(\sigma, \mathscr{L}) \subset \mathbf{I}_{\sigma}(\mathscr{L})$;

(b) $\mathscr{L}$ normal and complement generated implies $\mathbf{I}(\sigma, \mathscr{L}) \subset \mathbf{I}_{R}^{\sigma}(\mathscr{L})$;

(c) $\mathbf{I}_{R}^{\sigma}(\mathscr{L}) \subset \mathbf{I}(\sigma, \mathscr{L})$.

THEOREM 5.9. Consider $\mathbf{I}(\sigma, \mathscr{L})$ and $V(\sigma, \mathscr{L})$. Then $V(\sigma, \mathscr{L})$ is regular if and only if for all $\mu_{1}, \mu_{2} \in \mathbf{I}(\mathscr{L})$ and $v \in \mathbf{I}(\sigma, \mathscr{L})$, if $\mu_{1} \leq \mu_{2}, v(\mathscr{L})$ then $\mu_{2} \leq v(\mathscr{L})$.

Proof. For $\mu_{1}, \mu_{2} \in \mathbf{I}(\mathscr{L})$ we have $\underline{\mu}_{1}, \underline{\mu}_{2} \in \mathbf{I}\left(W_{\sigma}(\mathscr{L})\right)$ and then $\underline{\mu}_{1}, \underline{\mu}_{2} \in \mathbf{I}(V(\sigma, \mathscr{L}))$. If $V(\sigma, \mathscr{L})$ is regular then $S\left(\underline{\mu}_{1}\right)=S\left(\underline{\mu}_{2}\right)$, where $S\left(\underline{\mu}_{1}\right)=\cap\{V(\sigma, L) \in V(\sigma, \mathscr{L})$ | $\left.\underline{\mu}_{1}(V(\sigma, L))=1, L \in \mathscr{L}\right\}$. Let $v \in \mathbf{I}(\sigma, \mathscr{L}) ; \underline{v} \in \mathbf{I}(\sigma, V(\sigma, \mathscr{L}))$ and $\underline{\mu}_{1} \leq \underline{v}(V(\sigma, \mathscr{L}))$. Then $\nu \in S\left(\underline{\mu}_{2}\right)$, that is, $\mu_{2} \leq v(\mathscr{L})$.

Conversely, suppose that $\mu_{1}, \mu_{2} \in \mathbf{I}(\mathscr{L})$ and $\nu \in \mathbf{I}(\sigma, \mathscr{L})$, such that if $\mu_{1} \leq \mu_{2}, \nu(\mathscr{L})$ then $\mu_{2} \leq v(\mathscr{L})$. Let $\lambda_{1}, \lambda_{2} \in \mathbf{I}(V(\sigma, \mathscr{L}))$ and $\lambda_{1} \leq \lambda_{2}(V(\sigma, \mathscr{L}))$. Then $\lambda_{1}=\underline{\mu}_{1}$ and $\lambda_{2}=$ $\underline{\mu}_{2}$ with $\mu_{1}, \mu_{2} \in \mathbf{I}(\mathscr{L})$. Thus $\underline{\mu}_{1} \leq \underline{\mu}_{2}(V(\sigma, \mathscr{L}))$ which implies that $\mu_{1} \leq \mu_{2}(\mathscr{L})$, hence 
$S\left(\underline{\mu}_{2}\right) \subset S\left(\underline{\mu}_{1}\right)$. If $\lambda \in S\left(\underline{\mu}_{1}\right)$ then clearly $\lambda \in \mathbf{I}(\sigma, \mathscr{L})$ and $\mu_{1} \leq \lambda(\mathscr{L})$. By assumption, $\mu_{2} \leq \lambda(\mathscr{L})$ and then $\lambda \in S\left(\underline{\mu}_{2}\right)$. Hence $S\left(\underline{\mu}_{2}\right)=S\left(\underline{\mu}_{1}\right)$ and $V(\sigma, \mathscr{L})$ is regular.

THEOREM 5.10. Consider $\mathbf{I}(\sigma, \mathscr{L})$ and $V(\sigma, \mathscr{L})$. If $V(\sigma, \mathscr{L})$ is regular, then $\mathbf{I}(\sigma, \mathscr{L})=$ $\mathbf{I}_{R}^{\sigma}(\mathscr{L})$.

Proof. Let $\mu \in \mathbf{I}(\sigma, \mathscr{L})$, there exists $v \in \mathbf{I}_{R}(\mathscr{L})$ such that $\mu \leq v(\mathscr{L})$, hence $\mu \leq$ $\underline{\nu}(V(\sigma, \mathscr{L}))$ where $\underline{\mu} \in \mathbf{I}(\sigma, V(\sigma, \mathscr{L}))$ and $\underline{v} \in \mathbf{I}_{R}(V(\sigma, \mathscr{L})) . V(\sigma, \mathscr{L})$ is regular, therefore $S(\underline{\mu})=S(\underline{\nu})$, hence $v \leq \mu(\mathscr{L})$. It follows that $\mu=v(\mathscr{L})$ and since $v \in \mathbf{I}_{R}(\mathscr{L}), \mathbf{I}(\sigma, \mathscr{L}) \subset$ $\mathbf{I}_{\sigma}(\mathscr{L})$. Therefore $\mu \in \mathbf{I}_{R}(\mathscr{L}), \mathbf{I}_{\sigma}(\mathscr{L})$ and then $\mu \in \mathbf{I}_{R}^{\sigma}(\mathscr{L})$.

THEOREM 5.11. Consider $\mathbf{I}(\sigma, \mathscr{L})$ and $V(\sigma, \mathscr{L})$ with $\mathscr{L}$ Lindelöf. If for all $\mu_{1}, \mu_{2} \in \mathbf{I}(\mathscr{L})$ and $v \in \mathbf{I}(\sigma, \mathscr{L})$, such that if $\mu_{1} \leq \mu_{2}, v(\mathscr{L})$ then $\mu_{2} \leq v(\mathscr{L})$, it follows that $V(\sigma, \mathscr{L})$ is slightly and mildly normal.

Proof. By Theorem 5.9, $V(\sigma, \mathscr{L})$ is regular. As in Corollary 5.7, we show that $V(\sigma, \mathscr{L})$ is Lindelöf and then it is also slightly and mildly normal.

\section{REFERENCES}

[1] A. D. Alexandroff, Additive set-functions in abstract spaces, Rec. Math. [Mat. Sbornik] N. S. 8 (50) (1940), 307-342. MR 2,315c. Zbl 023.39701.

[2] G. Bachman and P.D. Stratigos, Lattice repleteness and some of its applications to topology, J. Math. Anal. Appl. 99 (1984), no. 2, 472-493. MR 85m:54020. Zbl 584.54019.

[3] G. Bachman and A. Sultan, Regular lattice measures: mappings and spaces, Pacific J. Math. 67 (1976), no. 2, 291-321. MR 58\#22476.

[4] G. Bachman and M. Szeto, On strongly measure replete lattices and the general Wallman remainder, Fund. Math. 122 (1984), no. 3, 199-217. MR 86d:28012. Zbl 567.28006.

[5] R. Connell, On certain Wallman spaces, Int. J. Math. Math. Sci. 17 (1994), no. 2, 273-276. MR 94j:28006. Zbl 809.28009.

[6] G. M. Eid, On normal lattices and Wallman spaces, Int. J. Math. Math. Sci. 13 (1990), no. 1, 31-38. MR 91d:28015. Zbl 714.28003.

[7] P.-S. Hsu, Applications of outer measures to separation properties of lattices and regular or $\sigma$-smooth measures, Int. J. Math. Math. Sci. 19 (1996), no. 2, 253-262. MR 97b:28003. Zbl 841.28005.

[8] M. Szeto, On normal lattices and separation properties of lattices, J. Indian Math. Soc. (N.S.) 58 (1992), no. 1-4, 51-64. MR 94h:06009. Zbl 883.06005.

[9] C. Vlad, On normal lattices and semiseparation of lattices, J. Indian Math. Soc. (N.S.) 56 (1991), no. 1-4, 259-273. MR 93f:06009b. Zbl 874.28019.

[10] H. Wallman, Lattices and topological spaces, Ann. of Math. 39 (1938), 112-126.

Carmen D. Vlad: Department of Mathematics, Pace University, New York, NY 10038, USA

E-mail address: cv1ad@pace.edu 


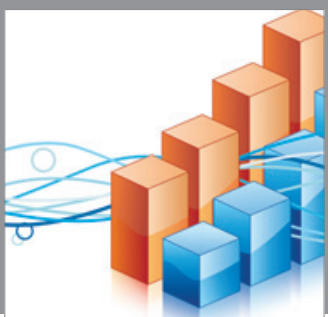

Advances in

Operations Research

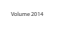

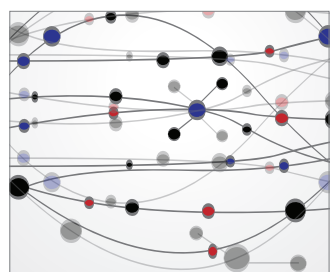

\section{The Scientific} World Journal
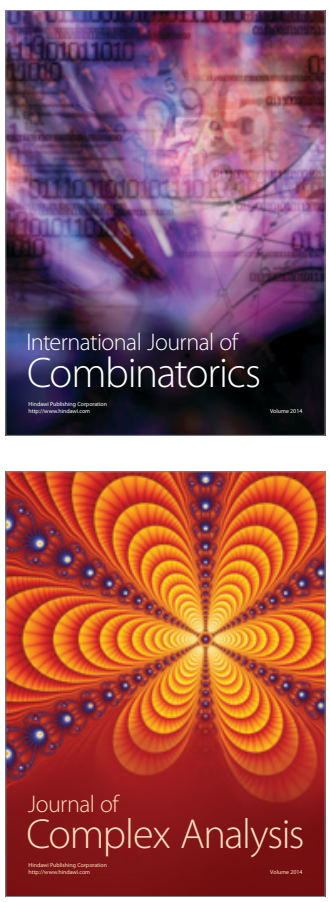

International Journal of

Mathematics and

Mathematical

Sciences
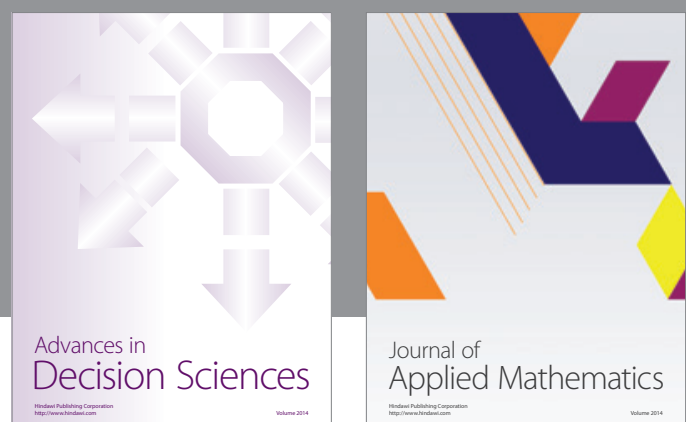

Journal of

Applied Mathematics
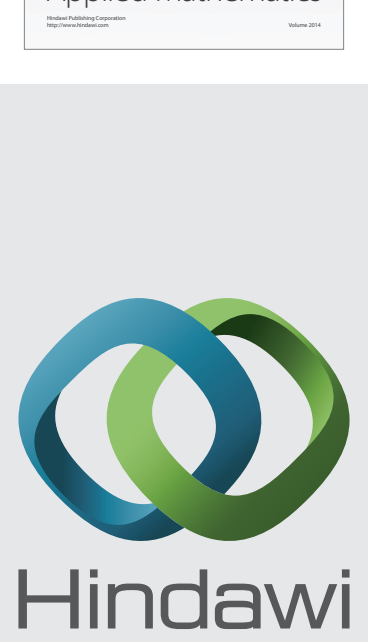

Submit your manuscripts at http://www.hindawi.com
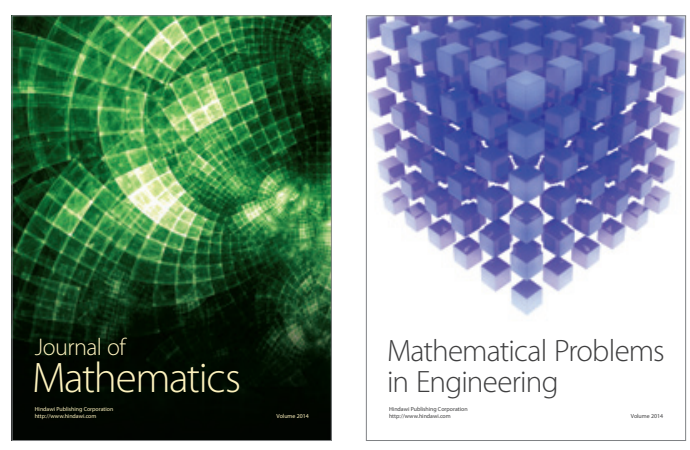

Mathematical Problems in Engineering
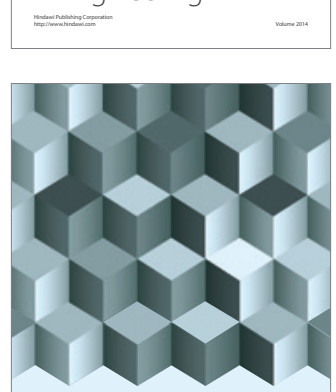

Journal of

Function Spaces
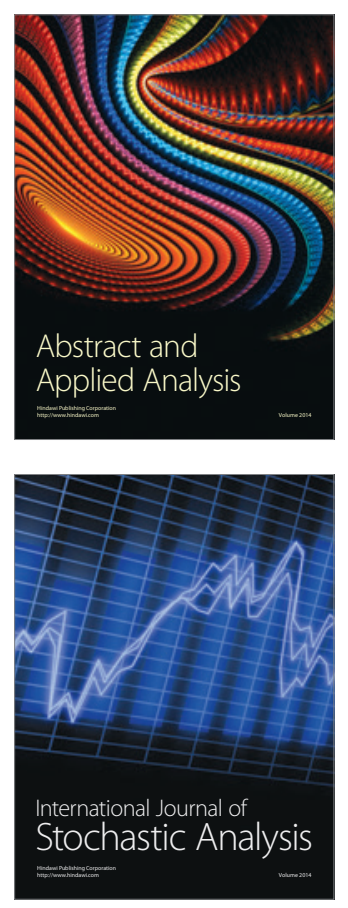

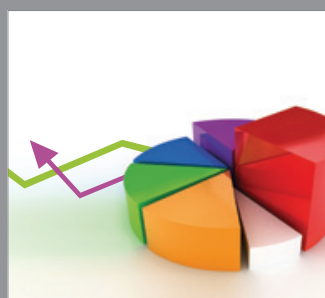

ournal of

Probability and Statistics

Promensencen
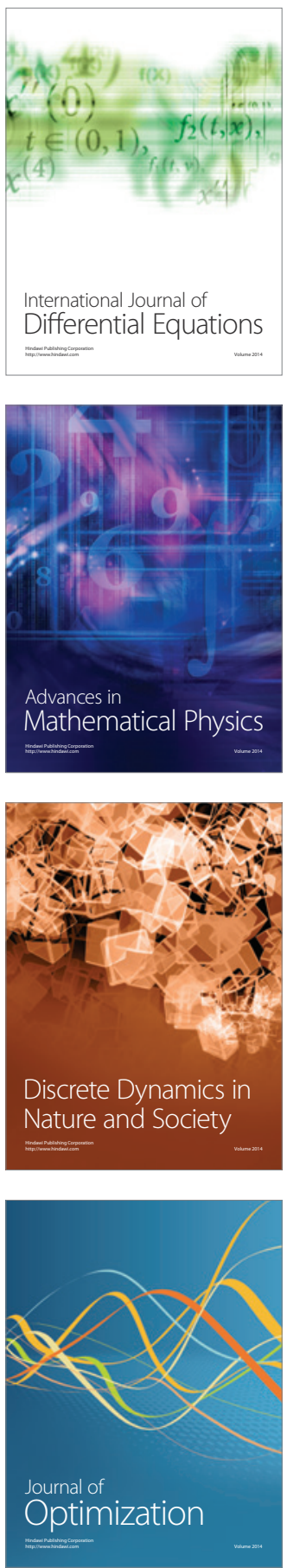